Meta

Journal des traducteurs

Translators' Journal

\title{
Pédagogie du traduire : les tâches cognitives de l'acte traductif
}

\section{Claude Tatilon}

Volume 52, numéro 1, mars 2007

Traductologie : une science cognitive

URI : https://id.erudit.org/iderudit/014731ar

DOI : https://doi.org/10.7202/014731ar

Aller au sommaire du numéro

\section{Éditeur(s)}

Les Presses de l'Université de Montréal

\section{ISSN}

0026-0452 (imprimé)

1492-1421 (numérique)

Découvrir la revue

\section{Citer cet article}

Tatilon, C. (2007). Pédagogie du traduire : les tâches cognitives de l'acte traductif. Meta, 52(1), 164-171. https://doi.org/10.7202/014731ar
Résumé de l'article

Comment comprend-on un énoncé écrit, comment construit-on une représentation mentale de ce qu'il dit, comment passe-t-on d'un énoncé donné d'une langue à un énoncé équivalent dans une autre langue - ces questions sont d'un intérêt brûlant pour la pédagogie de la traduction. J'ai tenté de leur trouver des réponses auprès des spécialistes des sciences cognitives, convaincu qu'en approfondissant la connaissance qu'on a de sa propre activité cognitive, on se prépare à mieux accomplir l'acte de traduction : savoir plus pour mieux traduire. C'est pourquoi il m'a semblé approprié d'élaborer un modèle qui donne une image nécessairement simplifiée mais néanmoins réaliste de l'activité mentale déployée lors du traduire, et ainsi de transformer l'acte traductif en objet sur lequel il soit possible d'avoir prise - en un outil de réflexion. 


\title{
Pédagogie du traduire: les tâches cognitives de l'acte traductif
}

\author{
CLAUDE TATILON \\ Université York, Toronto, Canada \\ tatilon@yorku.ca
}

\begin{abstract}
RÉSUMÉ
Comment comprend-on un énoncé écrit, comment construit-on une représentation mentale de ce qu'il dit, comment passe-t-on d'un énoncé donné d'une langue à un énoncé équivalent dans une autre langue - ces questions sont d'un intérêt brûlant pour la pédagogie de la traduction. J'ai tenté de leur trouver des réponses auprès des spécialistes des sciences cognitives, convaincu qu'en approfondissant la connaissance qu'on a de sa propre activité cognitive, on se prépare à mieux accomplir l'acte de traduction: savoir plus pour mieux traduire. C'est pourquoi il m'a semblé approprié d'élaborer un modèle qui donne une image nécessairement simplifiée mais néanmoins réaliste de l'activité mentale déployée lors du traduire, et ainsi de transformer l'acte traductif en objet sur lequel il soit possible d'avoir prise - en un outil de réflexion.
\end{abstract}

\begin{abstract}
How do we understand a written statement? How do we construct a mental representation of what it states? How do we switch from a statement in one language to another equivalent statement in another language? These questions are of paramount importance to the pedagogy of translation. I attempted to obtain answers to these questions from cognitive scientists, convinced that knowing more about our own cortical processes can improve the act of translation. The more we know, the better we can translate. I also thought it appropriate to develop a model that would provide us with a simplified, but nonetheless realistic, image of the mental activity that is involved during translation. This will therefore transform the translation process into an object we can grasp - a tool for reflection.
\end{abstract}

\section{MOTS-CLÉS/KEYWORDS}

pédagogie, information textuelle, compréhension, déverbalisation, représentation mentale

Dans Petites proses, recueil de Michel Tournier, ces quelques lignes: «Le mystère des plis du cerveau! À mesure que la neurologie avance dans la connaissance des douze milliards de cellules qui forment la substance grise, on voit s'épaissir ce mystère et progresser la conscience de notre ignorance, comme une lanterne descendue au bout d'une corde dans un gouffre n'en révèle que l'insondable profondeur. Le cerveau humain apparaît aujourd'hui comme un continent où presque tout est encore vierge, ses mers et ses îles, sa flore et sa faune, sa climatologie et son ethnographie. L'homme passe l'homme, disait Pascal ${ }^{1}$.»

L'extrême complexité du cerveau humain est attestée un peu partout dans les écrits des meilleurs spécialistes des sciences cognitives. Par exemple, chez Jean-Pierre Changeux, lors d'un dialogue avec le mathématicien Alain Connes: "Comme tu le sais, l'ensemble de notre encéphale se compose d'environ cent milliards de neurones, ce qui est, quand même, un chiffre élevé! Ces neurones sont reliés entre eux par des 
zones de contact discontinu ou synapses. Il y en a en moyenne environ dix mille par cellule nerveuse. Soit un nombre total de synapses, dans notre cerveau, de l'ordre de $10^{15}$. C'est un chiffre astronomique ${ }^{2}$.» Ailleurs, «tent[ant] de faire le point sur ce que nous enseignent, à ce stade, nos connaissances biologiques sur le cerveau et ses productions», Changeux admet "qu'il s'agit d'un état provisoire de notre savoir et que notre ignorance est immense ${ }^{3}$.»

Que pouvons-nous alors attendre, pour notre discipline, de ce type de recherches? L'acte traductif n'étant pas étranger à l'univers des réalités mentales, il n'est pas illégitime de nous poser la question - même si, devant un tel degré de difficulté, une attitude d'extrême prudence semble s'imposer: méfions-nous des incursions dans un domaine qui n'est pas le nôtre et qui ne saurait tolérer l'amateurisme. Néanmoins, je reste convaincu qu'en approfondissant la connaissance qu'on a de sa propre activité cognitive, on se prépare à mieux accomplir l'acte de traduction: savoir plus pour mieux traduire. C'est pourquoi il m’a semblé approprié d'élaborer un modèle qui donne une image nécessairement simplifiée mais néanmoins réaliste de l'activité mentale déployée lors du traduire, et ainsi de transformer l'acte traductif en objet sur lequel il soit possible d'avoir prise - en un outil de réflexion.

\section{Modèle de l'acte traductif}

L'acte traductif est le résultat de l'action conjuguée de trois systèmes organiques: le système visuel, le système significatif et le système émetteur ${ }^{4}$. Conformément aux faits accessibles à l'observation, on peut définir cet acte en disant qu'il consiste à reformuler par un texte d'arrivée (TA) dans une langue donnée ce qu'on a compris à la lecture d'un texte de départ (TD) écrit dans une autre langue, tout en précisant - autre évidence - qu'il constitue un processus allant du lu au compris, puis du compris au tra$d u i t^{5}$. L'acte traductif est donc d'abord onomasiologique, ensuite sémasiologique. Je propose de le modéliser ainsi:

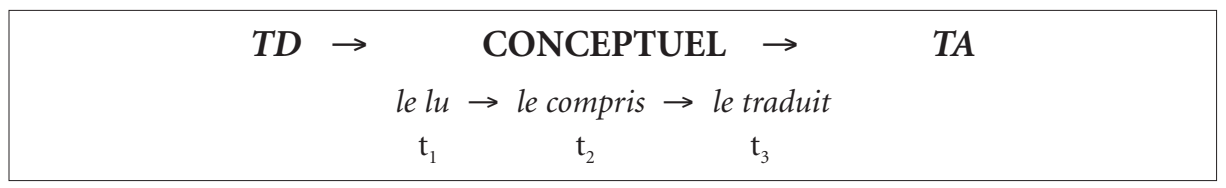

\section{Durée d'exécution de l'acte traductif}

Au moment de traduire (c'est-à-dire d'effectuer le passage de $t_{2}$ à $t_{3}$ ), j'ai simultanément sous les yeux du texte (TD) et à l'esprit du conceptuel (la compréhension que j'ai dégagée du texte par ma lecture). Me mettant alors à traduire, je constate:

1. Qu'il arrive que certains segments courts du TD se traduisent instantanément, sans aucun effort apparent, comme si ma pensée n'était point sollicitée; sitôt compris, ces segments font surgir à ma conscience leur équivalent en langue d'arrivée (LA). Il s'agit alors, surtout, d'unités lexicales ou de figements phraséologiques qui me sont familiers et dont j'ai mémorisé la traduction:

- mots communs aux deux lexiques: address, Marseille(s), Paris, tennis, typography;

- noms d'objets usuels: knife, fork, glass, napkin;

- termes appartenant à un domaine bien connu de moi: phoneme, syllable, stress, phrase, sentence, paragraph; 
- figements du genre: Nice to meet you. See you soon.

- ou encore, cette paire de prépositions anglaises immédiatement converties en noms français: From: $\square$ Expéditeur, To: $\square$ Destinataire.

Facilitée par des habitudes acquises de longue date, la traduction me paraît ici machinale: les segments se traduisent en bloc, automatiquement, comme si $t_{2}$ et $t_{3}$ ne faisaient qu'un. Appelons «transcodage» ce premier type de traduction où, à peine saisi, le sens «saute aux yeux/à l'esprit» pour se reformuler quasi instantanément.

2. Je constate aussi que, parfois, d'autres segments moins courts, qui sont le plus souvent des énoncés complets, sollicitent davantage ma pensée, mais qu'ils se traduisent néanmoins sans encombre, presque toujours «mot à mot», par simple conservation de la structure syntaxique. Mon effort de conceptualisation n'est alors pas très grand et la durée de $t_{3}$ à peine perceptible. Il s'agit cette fois d'une «traduction calquée» où chaque monème (ou presque) est analysé et traduit individuellement. C'est le cas de ces trois phrases: The sky is blue. At what time do you want to come? I'll do it tomorrow.

3. Je constate encore, beaucoup plus fréquemment, que d'autres segments, généralement assez longs, exigent un certain effort de reformulation, un certain labeur cognitif, souvent important. Une frontière est alors franchie: celle du raisonnement, de la «repensée» (ce «mot juste» est d'André Martinet). Même si, en $\mathrm{t}_{1}-\mathrm{t}_{2}$, le délestage du conceptuel s'est parfaitement effectué (ce qui semble être très souvent le cas), je dois cependant m'appesantir sur ce conceptuel issu de ma lecture, afin de le garder à l'abri de la langue d'arrivée (LD) et de lui éviter d'être repris par la forme initiale (qui constitue toujours un sérieux obstacle pour $\mathrm{t}_{3}$ ). Il me faut alors l'approfondir, le soumettre au raisonnement - le repenser - pour que puisse surgir à ma conscience la forme nouvelle qui me satisfera. Prenons deux exemples: I look forward to seeing you et Sincerely yours. Ainsi isolées, ces formules figées que l'on trouve en fin de lettre imposent, en français du moins, de choisir entre plusieurs variantes possibles en fonction de la situation de communication épistolaire. Et ce choix forcé interdit toute possibilité de traduction rapide.

Dans ces deux derniers exemples, la repensée est de nature, disons, pragmatique. Elle pourrait être aussi de nature culturelle, si le TD évoquait un référent insolite dans la culture d'arrivée. Comme dans cet exemple: Let's have a brunch together tomorrow. En effet, le mot-valise brunch (breakfast+lunch) désigne une pratique alimentaire peu connue dans l'Europe non anglophone en dehors de certains quartiers «branchés» des grandes villes. La solution se trouve alors dans la transposition: On se rencontre au petit déjeuner/ au petit dèj/ demain? Ou : On prend un café/ On casse une petite croûte/ demain, vers 10 heures? Voire: On déjeune (voire dîne pour le Midi de la France)/ ensemble, demain?

4. Je constate enfin, parfois, que certains segments du textes agissent comme des verroux et bloquent ma reformulation; c'est le cas de ces deux slogans publicitaires: Le lait, c'est vachement bon et Le parapluie Knirps 2000 vous plaira avant même qu'il ait plu! Dans ces cas de signifiance (je reviendrai plus bas sur cette notion), la traduction peut se faire attendre des jours et des jours, et même ne jamais advenir.

En résumé, lors d'un acte traductif, j'expérimente quatre types de reformulation du conceptuel:

1. Une reformulation quasi instantanée, comme s'il n'y avait aucun temps d'arrêt: $t_{2}$ semble ne pas exister - c'est le transcodage (À suivre $\square$ To be continued); 
2. Une reformulation rapide: $\mathrm{t}_{2}$ est très court, de l'ordre de la seconde - c'est la traduction calquée (The sky is blue $\square$ Le ciel est bleu);

3. Une reformulation ralentie: $\mathrm{t}_{2}$ a une durée relativement longue et très variable - c'est la traduction tout court, avec ses grandeurs et ses misères. Il faut alors bien remarquer que ce troisième type se distingue nettement des deux autres par un labeur cognitif plus important qui exige un raisonnement; il aboutit à une formulation courante.

4. Une reformulation entravée: $t_{2}$ exige maintenant, en plus d'un raisonnement, une analyse linguistique et aboutit à une invention verbale plus ou moins laborieuse: Milk. Udderly delicious! par exemple. Ou encore Knirps 2000. When it rains, it reigns!

\section{Incidences pédagogiques}

De ce qui précède, que déduire d'utile pour la pédagogie de la traduction? D’abord, une clarification des tâches respectives de l'interprète et du traducteur. Celle de l'interprète se limite aux trois premiers types de reformulation; le quatrième est exclu: les cas d'invention verbale sont manifestement rares dans le discours oral spontané et lorsqu'il en rencontre l'interprète se trouve le plus souvent désarmé. Si bien que l'essentiel de sa formation concerne la reformulation de type 3. En revanche, la formation du traducteur ne saurait négliger celle de type 4, les inventions verbales se rencontrant aussi, assez fréquemment, dans les textes utilitaires, destinés à la compréhension et à la représentation claires.

Il me semble aussi essentiel d'examiner le conceptuel qui est au centre du modèle et qui représente l'information que la personne qui traduit a su extraire de la lecture d'un TD - une information déverbalisée ${ }^{6}$, provisoirement retenue dans sa mémoire de travail et interprétée à l'aide des divers savoirs qui se trouvent stockés dans sa mémoire à long terme. Voici ce qu'en dit Bernard Pottier: «[...] il se construit quelque chose de conceptuel, qui est, je ne dis pas indépendant des langues - c'est un mot que je n'ai jamais employé - mais qui constitue un avant et un après du fonctionnement du langage: un avant quand je suis énonciateur, un après quand je suis récepteur. [...] Je serais incapable de répéter ce que vous avez dit avec les mêmes mots, avec les mêmes phrases, avec la même syntaxe, mais je peux conserver l'essentiel. Ce que je conserve est forcément de nature conceptuelle [...] Il est certain que, chaque fois que l'on parle de conceptualisation, la base est une expérience linguistique ${ }^{7}$.»

Un autre linguiste important, André Martinet, nous donne sur le conceptuel des précisions fort éclairantes: "puisque je parle français et que le mot maison est un mot français, je n'ai qu'à rechercher en moi-même ce que représente le mot maison, et je déterminerai ainsi le sens de ce mot. Malheureusement lorsque j'essaie de voir ce qu'il évoque pour moi, une image apparaît, plus ou moins composite, dont je suis sûr, par certains de ses traits, qu'elle n'est pas celle que le mot évoquera chez toute autre personne. Il est donc clair que cette image, qui d'ailleurs varie chez moi d'un instant à un autre, ne saurait être considérée comme le «sens» du mot, bien commun de tous les sujets de langue française. Tout ce que je sais du sens de maison, c'est qu'un certain type d'expérience est associé chez moi au signifiant/mezõ/ ou à son substitut graphique maison et que cette même association existe chez les autres personnes de langue française. [...] Il faut noter que la vue d'une maison ne déclenche pas automatiquement 
le processus linguistique qui lui est associé et que, parallèlement, l'emploi du mot maison n'entraîne pas nécessairement l'évocation d'une expérience vécue. Il est même vraisemblable qu'il n'y a rien de tel dans la plupart des cas et qu'un énoncé ne s'accompagne pas, en général, d'une série d'évocations ou de prises de conscience correspondant à chacune des unités significatives successives. Ceci ne serait guère compatible avec la rapidité du discours. [...] Un élément linguistique n’a réellement de sens que dans un contexte et une situation donnés; en soi, un monème ou un signe plus complexe ne comporte que des virtualités sémantiques dont certaines seulement se réalisent effectivement dans un acte de parole déterminé: pour reprendre l'exemple de maison, dans les actes de parole Madame n'est pas à la maison, il représente une maison de commerce, il lutta contre la Maison d'Autriche, le contexte fait apparaître dans chaque cas certaines virtualités et rejette les autres dans l'ombre ${ }^{8}$.»

Les spécialistes des sciences cognitives vont évidemment plus loin que les linguistes dans l'exploration du conceptuel, parlant de traces neuronales, d'images mentales, d'objets mentaux qui existent matériellement dans le cerveau, voire d'un système significatif ${ }^{9}$, et allant même jusqu'à confirmer l'existence de «cartes d'activation cérébrale» et d'une «géographie de la compréhension ${ }^{10}$. Pour eux, qui s'inscrivent résolument dans le courant matérialiste ${ }^{11}$, «l'image mentale ne doit pas être prise dans un sens évanescent ou immatériel, mais au contraire, comme une activité cérébrale et bien définie ${ }^{12} »$ : les représentations du langage prennent corps dans le cortex et sont donc un pur produit de l'activité cérébrale.

Dans un ouvrage au titre éloquent, L'empreinte des sens, le biologiste Jacques Ninio nous donne un exemple limpide d'image mentale ainsi que d'utiles précisions sur l'organisation de la mémoire humaine en de multiples compartiments: "Quelque part, dans une mémoire acoustique du cerveau, se trouve une représentation du mot bateau, qui permet de le repérer dans une conversation orale. Quelque part, dans une mémoire graphique, est représentée l'orthographe du mot. Autre part, dans une mémoire motrice, gutturale, sont représentés les ordres de commandes musculaires à accomplir pour prononcer «bateau». Ailleurs, dans d'autres mémoires, sont conservées des traces de perceptions se rapportant aux navires: le mélange caractéristique d'odeurs de cordage, de mazout, de peinture fraîche et d'air marin dans la mémoire olfactive, le hurlement des sirènes dans la mémoire acoustique, des images de bateaux dans la mémoire visuelle, des souvenirs de croisière dans la mémoire des épisodes » (p. 255). Un court passage qui en dit long sur l'origine de ces virtualités sémantiques dont parle Martinet et qui sont à la racine de nos associations individuelles et de nos connotations socialisées.

Méditer sur la sélection par le contexte de virtualités sémantiques particulières nous conduit directement à la notion d'information textuelle pertinente ${ }^{13}$ et à l'importance qu'elle revêt pour nos choix traductifs. Une importance indubitable pour la pédagogie de la traduction: cette notion soulage l'apprenant de la hantise de vouloir tout traduire du sens appréhendé et lui donne la lucidité nécessaire pour éviter de graves erreurs. Des erreurs du genre de celle-ci, rencontrée naguère dans la traduction française d'une encyclopédie américaine destinée à un jeune public. Pour illustrer la notion de synonymie, on y découvre un exemple pour le moins surprenant: petit $\leftrightarrow$ faible!! L'explication, c'est dans l'exemple anglais qu'on doit aller la chercher: small $\leftrightarrow$ little. On comprend alors qu'il s'agit d'une tentative, aussi désespérée qu'inutile, pour traduire un couple lexical qui n'a pas d'équivalent en langue française. Alors que 
n'importe quel autre couple - bateau $\leftrightarrow$ navire, soulier $\leftrightarrow$ chaussure - aurait pu faire l'affaire...

\section{Compréhension et reformulation}

Ajoutons un peu de complexité à notre modèle.

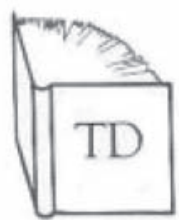

t1

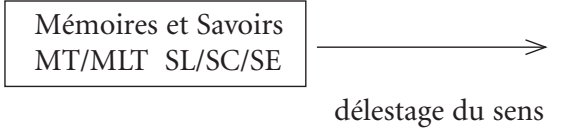

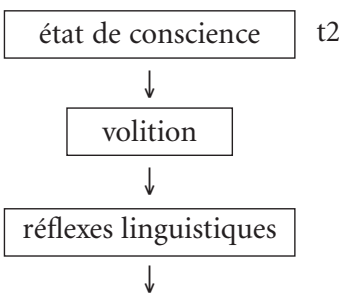

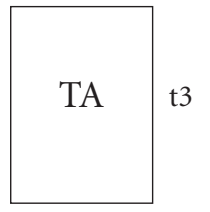

\section{Commentaires}

1. Mémoires et Savoirs. Lors de la lecture du traducteur, après que son système visuel aura conduit jusqu'à son cortex les signifiants du TD, ceux-ci iront se placer dans un espace de travail, «l'espace de travail neuronal ${ }^{14}$, qui est un milieu cortical où s'effectuent des opérations conscientes; puis, dans le temps très court de la mémoire dite de travail, le traducteur, au moyen des connaissances accumulées dans ses différentes mémoires à long terme, pourra déchiffrer l'information textuelle qu’ils contiennent. Ainsi, «la connaissance passée emmagasinée dans la mémoire à long terme sous forme de traces stables se réactualise en permanence dans le compartiment de travail, où elle est maintenue "en ligne" pendant que, par exemple, nous [déchiffrons un énoncé textuel] ${ }^{15}$ ». Dans le modèle, SL signifie «savoir linguistique», SC «savoir culturel» et SE «savoir encyclopédique». Devant, disons, un slogan publicitaire comme On a toujours besoin de petits pois chez soi, ce sont nos SL et SE qui nous permettent d'en saisir le sens manifeste, tandis que notre SC, qui nous en fournit l'intertexte (On a souvent besoin d'un plus petit que soi, La Fontaine), nous fait saisir le sens latent suggéré par le jeu verbal.

2. Délestage du sens. C'est le moment de la déverbalisation. Pédagogiquement parlant, il est important d'insister ici sur la nécessité d'écarter la «tyrannie de la forme» (Jean Darbelnet) qui fait constamment pièce à la déverbalisation et 
compromet l'idiomaticité de la reformulation. Marianne Lederer parle à ce propos de «la rémanence têtue du texte original», qu'elle commente ainsi: «les formes [du TD] veulent survivre à tout prix [et appellent] la recherche de correspondances directes qui s'opposent à la découverte d'équivalences satisfaisantes ${ }^{16}{ }^{\prime}$.

3. État de conscience. C'est le conceptuel, dont il a été largement question ci-dessus.

4. Volition. La volonté comme déclencheur des réflexes linguistiques... les émotions, la motivation, le désir de traduire, «le travail dans la joie» comme facilitateurs de l'acte traductif - nous nous trouvons ici sur un terrain miné. Le neurologue américain Antonio Damasio avance une théorie selon laquelle les émotions permettraient un «marquage» cortical qui influencerait nos actions de façon déterminante; un marquage positif agirait alors comme un signal d'encouragement. Quant au philosophe Paul Ricœur, il nous encourage à faire notre deuil de l'idéal de traduction parfaite, afin de pouvoir accommoder à la fois l'étranger et le domestique et de nous soustraire ainsi à l'angoisse ressentie devant l'inaccessible identité de sens. Sa recette: l'hospitalité langagière, «où le plaisir d'habiter la langue de l'autre est compensé par le plaisir de recevoir chez soi, dans sa propre demeure d'accueil, la parole de l'étranger ${ }^{17}{ }^{\prime}$.

5. Réflexes linguistiques. C'est ici le dernier stade de l'acte traductif: celui de la reformulation. Bernard Pottier en propose un schéma décomposé en quatre stades $^{18}$ qui complémente notre modèle. Après une «mise en scène» correspondant à la déverbalisation, il postule «une mise en signes» qui correspond à la recherche des unités lexicales appropriées, une «mise en schème» qui correspond aux monèmes syntaxiques (de temps, d'aspect, de fonction) et, en dernier lieu, une "mise en chaîne» qui ajoute les déterminants obligatoires et complète l'énoncé. Soit l'exemple ci-dessous:

\section{The bus just arrived at the station.}

«mise en scène»: contenu déverbalisé de l'énoncé

«mise en signes» : gare autobus arriver

«mise en schème»: autobus arriver/passé proche/ à gare (les fonctions sont assumées ici par l'ordre des mots et par la préposition)

«mise en chaîne»: L'autobus vient d'arriver à la gare.

Comment comprenons-nous un énoncé écrit, comment construisons-nous une représentation mentale de ce qu'il dit, comment passons-nous d'un énoncé donné d'une langue à un énoncé équivalent dans une autre langue - ces questions à la frontière de la biologie et de la linguistique restent d'un intérêt brûlant. Le modèle ici proposé a tenté d'en sérier quelques-unes, pour lesquelles les réponses prudentes ou risquées des spécialistes, et sans doute vouées à demeurer incomplètes, ne sont pas toujours totalement satisfaisantes du point de vue qui est le nôtre. Cependant, même incomplètes, ces savantes réponses paraissent susceptibles d'enrichir notre pédagogie et d'améliorer notre savoir-faire.

\section{NOTES}

1. Petites proses, «Maître Cerveau», p. 95-96.

2. Matière à pensée, p. 127.

3. L'Homme de vérité, p. 322.

4. À propos du «système significatif» (appelé encore esprit, pensée, conscience) que les partisans du dualisme préfèrent concevoir comme du spirituel à l'état pur, voir Les réflexes linguistiques de Carlos 
Ramirez de la Lastra et Miguel Garcia Vives. Ces auteurs écrivent, p. 20: «Si les fonctions de l'audition [ou de la vision] et de la prononciation occupent des espaces corticaux bien délimités, il est logique de supposer que la signification de ce que l'homme entend [ou lit] ou prononce occupe aussi un espace dans le cortex.»

5. J.-R. Ladmiral, avec la fantaisie néologique qu'on lui connaît, parle du «no man's langue», qui s'étend $\mathrm{du}$ «déjà-plus du texte-source» au "pas-encore du texte-cible.» (La linguistique, "Linguistique et Traductologie», p. 40.)

6. Du «no-man's langue», selon le mot de Ladmiral, cité plus haut. Voir, dans le même article, ce que cet auteur dit du concept de déverbalisation tel que l'ont défini Danica Seleskovitch et Marianne Lederer.

7. «Débat avec Bernard Pottier», La linguistique, p. 145.

8. Éléments de linguistique générale, p. 34-36.

9. Supra, note 4.

10. Jean-Pierre Changeux, La nature et la règle, p. 123.

11. «Je défends [...] une épistémologie matérialiste forte, la seule qui me paraisse acceptable de la part d'un scientifique averti, soucieux de cohérence», déclare Changeux dans Matière à pensée (titre évocateur), à la page 46.

12. Changeux, ibid., p. 111. On lira aussi avec profit le compte rendu de L'homme neuronal, de Jean-Pierre Changeux, par Georges Mounin, publié dans la revue Diogène, p. 49-71. Et aussi celui d'Anne Szulmajster-Celnikier, "Quelques réflexions sur la linguistique et les neurosciences, à propos de L'Homme de vérité de Jean-Pierre Changeux», paru dans La linguistique 40, p. 177-191.

13. Voir mon Traduire, en particulier le chapitre VIII traitant de «La pertinence communicative».

14. Jean-Pierre Changeux, L'Homme de vérité, p. 136-145, et La nature et la règle, p. 155-159.

15. Jean-Pierre Changeux, ibid., p. 160.

16. «La théorie interprétative de la traduction», Le français dans le monde, p. 15. À propos de cette théorie, sans vouloir en diminuer la portée pédagogique, j’insisterai tout de même sur le fait qu'elle reste muette sur les formes stylistiques d'un texte, celles qui contribuent efficacement à sa signification ou à la délectation esthétique qu'il est susceptible de provoquer.

17. Sur la traduction, p. 20.

18. Sémantique générale, p. 18.

\section{RÉFÉRENCES}

Changeux, J.-P. et A. Connes (1992): Matière à pensée, Paris, Éditions Odile Jacob.

Changeux, J.-P. et P. Ricceur (1998): La nature et la règle. Ce qui nous fait penser, Paris, Éditions Odile Jacob.

Changeux, J.-P. (2002): L'Homme de vérité, Paris, Éditions Odile Jacob.

Ladmiral, J.-R. (2004) : «Dichotomies traductologiques», Paris, PUF, La linguistique (numéro spécial Linguistique et traductologie) 40-1.

Lederer, M. (1987): «La théorie interprétative de la traduction», Le français dans le monde (numéro spécial Retour à la traduction), p. 11-17.

Martinet, A. (1991): Éléments de linguistique générale, Paris, Armand Colin, (3e édit.,1970, 1980).

Mounin, G. (1992): "Sémantique et neurologie: L’homme neuronal et la linguistique », Diogène 157, p. 49-71.

Ninio, J. (1991): L'empreinte des sens, Paris, Éditions Odile Jacob.

Pottier, B. (1992): Sémantique générale, Paris, PUF.

Pottier, B. (1996): «Débat avec Bernard Pottier», La linguistique 32-1, Paris, PUF.

Ramirez de la Lastra, C. et M. Garcia Vives (1981): Les réflexes linguistiques (traduit de l'espagnol par A. GonZalez-Rivero), Paris, PUF.

Ricceur, P. (2004): Sur la traduction, Paris, Bayard.

Szulmajster-Celnikier, A, (2004) : "Quelques réflexions sur la linguistique et les neurosciences, à propos de L'Homme de vérité de Jean-Pierre Changeux», La linguistique 40-1, p. 177-191.

Tatilon, C. (1986): Traduire. Pour une pédagogie de la traduction, Toronto, Éditions du GREF.

Tournier, M. (1986): Petites proses, Paris, Éditions Gallimard. 